\title{
OSCILLATIONS IN CERTAIN DIFFERENCE EQUATIONS
}

\author{
ZDZISŁAW SZAFRAŃSKI AND BLAŻEJ SZMANDA
}

\begin{abstract}
We obtain sufficient conditions for the oscillation of all solutions of some linear difference equations with variable coefficients.
\end{abstract}

\section{Introduction}

Recently, there has been a lot of interest in studying the oscillation of the solutions of difference equations. See for example [2,3], [5], [7]-[10], [12]-[16] and Chapter 7 in recent book by Györi and Ladas [4]. For the general theory of difference equations the reader is referred to the recent books $[1,6,11]$.

Our purpose in this paper is to give in general form the sufficient conditions for the oscillation of some linear difference equations. The results obtained here include and generalize those in [7] and [12].

In this paper we are concerned with a class of linear difference equations of the form

$$
\Delta\left(y_{n}+p y_{n-k}\right)+\sum_{i=1}^{m} p_{i}(n) y_{n-k_{i}}=0, \quad n=0,1, \ldots
$$

where $\Delta$ denotes the forward difference operator: $\Delta x_{n}=x_{n+1}-x_{n}$ for any sequence $\left(x_{n}\right)$ of real numbers, $\left(p_{i}(n)\right) \quad(i=1, \ldots, m)$ are sequences of nonnegative numbers, $p \neq 0$, and $k_{i} \quad(i=1, \ldots, m)$ are integers such that $0<k_{1}<k_{2}<\cdots<k_{m}, k$ is a positive integer. The sequences $\left(p_{i}(n)\right)(i=1, \ldots, m)$ are supposed to be not identically zero.

Let $K=\max \left\{k, k_{m}\right\}$. Then by a solution of Eq. (1) we mean a sequence $\left(y_{n}\right)$ which is defined for $n \geq-K$ and which satisfies (1) for all large $n$. A nontrivial solution $\left(y_{n}\right)$ of (1) is said to be oscillatory if for every $N>0$ there exists an $n \geq N$ such that $y_{n} y_{n+1} \leq 0$. Otherwise it is called nonoscillatory. Equation (1) is said to be oscillatory if all its nontrivial solutions are oscillatory.

Received April 12, 1995.

1991 Mathematics Subject Classification. 39A10.

Key words and phrases. Oscillations, difference equations, difference inequality. 


\section{Some Lemmas}

In this section we give some lemmas which will be useful in our study of Eq. (1).

Lemma 1. Let the sequences $\left(p_{i}(n)\right)(i=1, \ldots, m)$ be periodic with period $k$ and $\left(y_{n}\right)$ is any solution of Eq. (1). Let us denote

$$
z_{n}=y_{n}+p y_{n-k}, \quad w_{n}=z_{n}+p z_{n-k}
$$

for all large $n$.

Then the sequences $\left(z_{n}\right)$ and $\left(w_{n}\right)$ are the solutions of (1) for all large $n$.

Proof. It suffices to show that $\left(z_{n}\right)$ is a solution of (1). Denote the left hand side of (1) by $L\left(y_{n}\right)$. Then we have

$$
\begin{aligned}
L\left(z_{n}\right) & =\Delta z_{n}+p \Delta z_{n-k}+\sum_{i=1}^{m} p_{i}(n) z_{n-k_{i}} \\
& =\Delta\left(y_{n}+p y_{n-k}\right)+p \Delta\left(y_{n-k}+p y_{n-2 k}\right)+\sum_{i=1}^{m} p_{i}(n)\left(y_{n-k_{i}}+p y_{n-k_{i}-k}\right) \\
& =\Delta\left(y_{n}+p y_{n-k}\right)+\sum_{i=1}^{m} p_{i}(n) y_{n-k_{i}}+p\left\{\Delta\left(y_{n-k}+p y_{n-2 k}\right)+\sum_{i=1}^{m} p_{i}(n) y_{n-k_{i}-k}\right\} \\
= & p\left\{\Delta\left(y_{n}+p y_{n-k}\right)+\sum_{i=1}^{m} p_{i}(n+k) y_{n-k_{i}}\right\}=0
\end{aligned}
$$

Lemma 2. Let $-1 \leq p<0$ and $\left(y_{n}\right)$ be an eventually positive solution of (1). Then $\left(z_{n}\right)$ defined by $(2)$ is an eventually positive nonincreasing sequence.

In addition, if the sequences $\left(p_{i}(n)\right)(i=1, \ldots, m)$ are periodic with period $k$ then the sequence $\left(w_{n}\right)$ defined by $(2)$ is an eventually positive nonincreasing solution of Eq. (1).

Proof. From Eq. (1) we have

$$
\Delta z_{n}=-\sum_{i=1}^{m} p_{i}(n) y_{n-k_{i}} \leq 0 \text { for all lage } n
$$

Hence $\left(z_{n}\right)$ is an eventually positive or eventually negative sequence.

Suppose that

$$
z_{n} \leq z_{n_{0}}<0 \text { for } n \geq n_{0} .
$$

Since $-1 \leq p<0$, we have

$$
y_{n} \leq-p y_{n-k}+z_{n_{0}} \leq y_{n-k}+z_{n_{0}}
$$


and for $n=n_{0}+k i, i=1,2, \ldots$ we get

$$
y_{n_{0}+k i} \leq y_{n_{0}+k(i-1)}+z_{n_{0}} .
$$

From the above inequalities we obtain

$$
y_{n_{0}+i k} \leq i z_{n_{0}}+y_{n_{0}}
$$

By letting $i \rightarrow \infty$, we note that $y_{n_{0}+i k}$ will be negative which is a contradiction with $y_{n}>0$ eventually. Thus $\left(z_{n}\right)$ is an eventually positive sequence and by Lemma 1 is an eventually positive solution of (1). Then we have

$$
\Delta w_{n}=-\sum_{i=1}^{m} p_{i}(n) z_{n-k_{i}} \leq 0 \text { for all large } n .
$$

Since $\lim _{n \rightarrow \infty} z_{n}=\alpha \geq 0$, hence from (2) we get

$$
\lim _{n \rightarrow \infty} \bar{w}_{n}=(1+p) \alpha \geq 0
$$

Therefore $w_{n}>0$ for all large $n$ and our assertion is true.

Lemma 3. Let $p<-1$ and there is an index $j \in\{1, \ldots, m\}$ such that $\sum^{\infty} p_{j}(n)=\infty$. If $\left(y_{n}\right)$ be an eventually positive solution of $(1)$, then $\left(z_{n}\right)$ defined by (2) is an eventually negative nonincreasing sequence.

In addition, if the sequences $\left(p_{i}(n)\right)(i=1, \cdots, m)$ are periodic with period $k$, then the sequence $\left(w_{n}\right)$ defined by $(2)$ is an eventually positive nondecreasing solution of $E q$. (1)

Proof. Similarly as in the proof of Lemma 2, we see that $\left(z_{n}\right)$ is a nonincreasing sequence for all large $n$. Now we show that $z_{n}<0$ eventually.

If not, then we have

$$
y_{n}>-p y_{n-k} \text { for } n \geq n_{0}
$$

i.e.

$$
0<y_{n-k}<-\frac{1}{p} y_{n} \text { for } n \geq n_{0},
$$

which implies

$$
0<y_{n-k}<\left(-\frac{1}{p}\right)^{i} y_{n+(i-1) k}, \quad i=1,2, \ldots, \quad n \geq n_{0} .
$$

Since $\left(-\frac{1}{p}\right)^{i} \rightarrow \infty$, as $i \rightarrow \infty$, hence from (3) follows that $y_{n} \rightarrow \infty$ as $n \rightarrow \infty$. 
From Eq. (1) we get

$$
\Delta z_{n}=-\sum_{i=1}^{m} p_{i}(n) y_{n-k_{i}} \leq-p_{j}(n) y_{n-k_{j}} \leq-M p_{j}(n)
$$

for all large $n$, say for $n \geq n_{1}$, where $M$ is a positive number. Summing (4) we have

$$
z_{n+1}-z_{n_{1}} \leq-M \sum_{i=n_{1}}^{n} p_{j}(i)
$$

which implies that $z_{n} \rightarrow-\infty$ as $n \rightarrow \infty$. This contradicts the fact that $z_{n}>0$ for $n \geq n_{0}$. Thus by Lemma 1 and the above proof $\left(z_{n}\right)$ is a negative solution of (1).

Therefore we have

$$
\Delta w_{n}=-\sum_{i=1}^{m} p_{i}(n) z_{n-k_{i}} \geq 0 \text { for all large } n .
$$

We show that $w_{n}>0$ eventually. Suppose that

$$
w_{n}=z_{n}+p z_{n-k}<0 \quad \text { for all } n \geq n_{0}
$$

i.e.

$$
-\frac{1}{p} z_{n}<z_{n-k}<0 \text { for } n \geq n_{0}
$$

which implies

$$
\left(-\frac{1}{p}\right)^{i} z_{n+(i-1) k}<z_{n-k}<0, \quad n \geq n_{0}, \quad i=1,2, \cdots .
$$

Since $\left(-\frac{1}{p}\right)^{i} \rightarrow 0$ as $i \rightarrow \infty$, hence from (6) follows that $z_{n} \rightarrow-\infty$ as $n \rightarrow \infty$.

From (5) we have for all large $n$, say for $n \geq n_{1}$

$$
\Delta w_{n}=-\sum_{i=1}^{m} p_{i}(n) z_{n-k_{i}} \geq-p_{j}(n) z_{n-k_{j}} \geq M p_{j}(n)
$$

where $M$ is a positive constant. Summing the above inequalities we get

$$
w_{n+1}-w_{n_{1}} \geq M \sum_{i=n_{1}}^{n} p_{j}(i) \rightarrow \infty \text { as } n \rightarrow \infty,
$$

that is $w_{n} \rightarrow \infty$ as $n \rightarrow \infty$, which contradicts the fact that $w_{n}<0$ for $n \geq n_{0}$. Thus the proof is complete. 


\section{Main Results}

Now we establish the oscillation results for Eq. (1) according to the values of $p$. Further we assume that the sequences $\left(p_{i}(n)\right)(i=1, \ldots, m)$ are periodic with period $k$ and denote

$$
p(n)=\sum_{i=1}^{m} p_{i}(n), \quad n=0,1, \ldots
$$

Theorem 1. Let $-1<p<0$. If the inequality

$$
\Delta x_{n}+\frac{1}{1+p} p(n) x_{n-k_{1}} \leq 0, \quad n=0,1,2, \ldots,
$$

has no eventually positive solution, then the equation (1) is oscillatory.

Proof. If not, we assume without loss of generality that $\left(y_{n}\right)$ is an eventually positive solution. Then, by Lemma 2 , for the sequence $\left(z_{n}\right)$ and $\left(w_{n}\right)$ defined by $(2)$ we have

$$
z_{n}>0, \quad \Delta z_{n} \leq 0 \text { and } w_{n}>0, \quad \Delta w_{n} \leq 0
$$

for all large $n$. Therefore we get

$$
w_{n}=z_{n}+p z_{n-k} \leq(1+p) z_{n}
$$

that is

$$
z_{n} \geq \frac{w_{n}}{1+p}
$$

By Lemmas 1,2 and the above inequality we may write

$$
\Delta w_{n}=-\sum_{i=1}^{m} p_{i}(n) z_{n-k_{i}} \leq-\sum_{i=1}^{m} p_{i}(n) z_{n-k_{1}} \leq-p(n) \frac{w_{n-k_{1}}}{1+p}
$$

for all large $n$.

Therefore $\left(w_{n}\right)$ is an eventually positive solution of (7), which is impossible. The proof is complete.

Theorem 2. Let $p=-1$. If the inequality

$$
\Delta x_{n}+p(n) x_{n-k_{1}} \leq 0, \quad n=0,1,2, \ldots,
$$

has no eventually positive solution, then the equation (1) is oscillatory.

Proof. The proof of this theorem is essentially the same as the proof of Theorem (1), and hence is omitted. 
Remark. It is worth to note that if (8) has no eventually positive solution, then (7) has no eventually positive solution.

In the next case we also assume a stronger condition than in Theorem 1.

Theorem 3. Let $p>0$ and $k<k_{1}$. If the inequality

$$
\Delta x_{n}+\frac{1}{1+p} p(n) x_{n-\left(k_{1}-k\right)} \leq 0, \quad n=0,1,2, \ldots
$$

has no eventually positive solution, then the equation (1) is oscillatory.

Proof. Let $\left(y_{n}\right)$ be an eventually positive solution of (1). Then for the sequences $\left(z_{n}\right)$ and $\left(w_{n}\right)$ defined by $(2)$ in view of Lemma 1 we have

$$
z_{n}>0, \Delta z_{n} \leq 0 \text { and } w_{n}>0, \Delta w_{n} \leq 0 \text { eventually. }
$$

Therefore

$$
w_{n}=z_{n}+p z_{n-k} \leq(1+p) z_{n-k} .
$$

Since $\left(z_{n}\right)$ is a nonincreasing solution of (1), hence

$$
\Delta w_{n}=-\sum_{i=1}^{m} p_{i}(n) z_{n-k_{i}} \leq-\sum_{i=1}^{m} p_{i}(n) z_{n-k_{1}}
$$

and, by $(10)$, we get

$$
\Delta w_{n} \leq-p(n) \frac{w_{n-k_{1}+k}}{1+p}
$$

for all large $n$. Thus $\left(w_{n}\right)$ is an eventually positive solution of (9). This contradiction proves the Theorem.

Theorem 4. Let $p<-1, k\rangle k_{m}$ and there exists an index $j \in\{1, \ldots, m\}$ such that $\sum^{\infty} p_{j}(n)=\infty$. If the inequality

$$
\Delta x_{n}+\frac{1}{1+p} p(n) x_{n+k-k_{m}} \geq 0, \quad n=0,1,2, \ldots
$$

has no eventually positive solution, then the equation (1) is oscillatory.

Proof. If not, let $\left(y_{n}\right)$ be an eventually positive solution of (1). By Lemma 3 , for the sequences $\left(z_{n}\right)$ and $\left(w_{n}\right)$ defined by (2) we have

$$
z_{n}<0, \Delta z_{n} \leq 0 \text { and } w_{n}>0, \Delta w_{n} \geq 0 \text { eventually. }
$$

Note that

$$
w_{n} \leq(1+p) z_{n-k}
$$


and thus

$$
z_{n-k} \leq \frac{w_{n}}{1+p} \text { for all large } n
$$

Since $\left(z_{n}\right)$ is a nonincreasing solution of (1), hence

$$
\Delta w_{n}=-\sum_{i=1}^{m} p_{i}(n) z_{n-k_{i}} \geq-\sum_{i=1}^{m} p_{i}(n) z_{n-k_{m}},
$$

and in view of (12) we get

$$
\Delta w_{n} \geq-\sum_{i=1}^{m} p_{i}(n) \frac{w_{n+k-k_{m}}}{1+p}
$$

thus $\left(w_{n}\right)$ is an eventually positive solution of (11), a contradiction.

In the last Theorem we does not assume that the sequences $\left(p_{i}(n)\right)(i=1, \ldots, m)$ are periodic.

Theorem 5. Let $p\langle-1, k\rangle k_{m}$ and there exists an index $j \in\{1, \ldots, m\}$ such that $\sum^{\infty} p_{j}(n)=\infty$. If the inequality

$$
\Delta x_{n}+\frac{1}{p} p(n) x_{n+k-k_{m}} \leq 0, \quad n=0,1, \ldots
$$

has no eventually negative solution, then the equation (1) is oscillatory.

Proof. If not, assume that there is an eventually positive solution $\left(y_{n}\right)$ of (1). Then, by Lemma 3 , for the sequence $\left(z_{n}\right)$ defined by (2) we have

$$
z_{n}<0 \text { and } \Delta z_{n} \leq 0 \text { eventually. }
$$

Evidently

$$
z_{n}=y_{n}+p y_{n-k}>p y_{n-k}
$$

i.e.

$$
y_{n-k}>\frac{1}{p} z_{n} \quad \text { eventually. }
$$

From (1) and (14) we get

$$
\Delta z_{n}=-\sum_{i=1}^{m} p_{i}(n) y_{n-k_{i}} \leq-\frac{1}{p} \sum_{i=1}^{m} p_{i}(n) z_{n+k-k_{i}}
$$

and by monotonicity of $\left(z_{x_{c}}\right)$, we obtain

$$
\Delta z_{n} \leq-\frac{1}{p} \sum_{i=1}^{m} p_{i}(n) z_{n+k-k_{m}}
$$


i.e. $\left(z_{n}\right)$ is an eventually negative solution of (13), which is impossible. This completes the proof.

From the above theorems one can obtain some effective oscillation criteria for difference equation of the form (1). Namely, each condition which guarantee that the suitable difference inequality appearing in Theorem 1-5 cannot have eventually positive or eventually negative solutions, gives oscillation criterion for equation (1).

For example, by Theorem 7.6 .1 in [4] (comp. [10]) we obtain oscillation criterion from Theorem 3, which improves and generalizes Th. 4 of [7] and Th. 5.1 of [12].

Corollary. Assume that $p>0, k<k_{1}$ and $p_{i}(n) \geq 0(i=1, \ldots, m)$ are periodic with period $k$.

If

$$
\frac{1}{1+p} \liminf _{n \rightarrow \infty} \sum_{i=n-k_{1}+k}^{n-1} \sum_{j=1}^{m} p_{j}(i)>\left(\frac{k_{1}-k}{k_{1}-k+1}\right)^{k_{1}-k+1},
$$

then every solution of (1) oscillates.

The above Corollary remains true, if we replace the condition (15) by the following (comp. [2], [14])

$$
\limsup _{n \rightarrow \infty} \sum_{i=n-k_{1}+k}^{n} \sum_{j=1}^{m} p_{j}(i)>1+p .
$$

Analogous corollaries which improve and generalize other results of [7] and [12] one can be obtained from the remaining theorems of this paper.

\section{References}

[1] R. P. Agarwal, Difference Equations and Inequalities. Theory, Methods and Applications, Marcel Dekker, New York, 1992.

[2] L. H. Erbe and B. G. Zhang, "Oscillation of discrete analogues of delay equation," Diff. Int. Equat., 2(1989), 300-309.

[3] D. A. Georgiou, E. A. Grove and G. Ladas, "Oscillations of neutral difference equation," Appl. Anal., 33(1989), 243-253.

[4] I. Györi and G. Ladas, Oscillation Theory of Delay Differential Equations with Applications, Oxford, 1991.

[5] J. W. Hooker and W. T. Patula, "A second order nonlinear equation: Oscillation and asymptotic behaviour," J. Math. Anal., 91(1983), 9-29.

[6] W. G. Kelley and A. C. Peterson, Difference Equations an Introduction with Applications, Acad. Press, Inc. Boston, 1991.

[7] J. Korczak and J. Popenda, "Oscillatory and nonoscillatory solutions of difference equations," An. St. Univ. "AL. I. Cuza" Iasi, 36(1990), 361-370.

[8] G. Ladas, "Explicit conditions for the oscillation of difference equations, " J. Math. Anal. Appl., 153(1990), 276-287.

[9] G. Ladas, Ch. G. Philos and Y. G. Sficas, "Necessary and sufficient conditions for the oscillation of difference equations," Libertas Math. 9(1989), 121-125. 
[10] G. Ladas, Ch. G. Philos and Y. G. Sficas, "Sharp conditions for the oscillation of delay difference equations," J. Applied Math. and Simulation, 2(1989), 101-112.

[11] V. Lakshmikantham and D. Trigiante, Theory of Difference Equations, Numerical Methods and Applications, Acad. Press, Inc. New York - London, 1988.

[12] B. S. Lalli, B. G. Zhang and J. Z. Li, "On the oscillation of solutions and existence of positive solutions of neutral difference equations," J. Math. Anal. Appl. 158(1991), 213-233.

[13] B. S. Lalli, B. G. Zhang, "On existence of positive solutions and bounded oscillations for neutral difference equations," J. Math. Anal. Appl., 166(1992), 272-287.

[14] Z. Szafrański and B. Szmanda, "A note on the oscillation of some difference equations," Fasc. Math., 21(1990), 57-63.

[15] B. Szmanda, "Oscillatory behaviour of certain difference equations," Fasc. Math., 21(1990), 65-78.

[16] A. Wyrwińska, "Oscillations of solutions of difference equations," Rad. Mat., 6(1990), 41-48, 1990.

Institute of Mathematics, Poznań University of Technology, 60-965 Poznań, Poland. 